\title{
Designing the Audience Journey through Repeated Experiences
}

\author{
Steve Benford ${ }^{1}$, Chris Greenhalgh${ }^{1}$, Adrian Hazzard ${ }^{1}$, Alan Chamberlain ${ }^{1}$, Maria Kallionpää², \\ David M. Weigl ${ }^{3}$, Kevin R. Page ${ }^{3}$ and Mengdie Lin ${ }^{4}$ \\ ${ }^{1}$ Mixed Reality Lab, University of Nottingham, Nottingham, UK \\ \{firstname.lastname\}@nottingham.ac.uk \\ ${ }^{2}$ Department of Communication and Psychology, Aalborg University, Aalborg, Denmark \\ kallionpaa@hum.aau.dk \\ ${ }^{3}$ Oxford e-Research Centre, University of Oxford, Oxford, UK \{firstname.lastname\}@oerc.ox.ac.uk \\ ${ }^{4}$ School of Information, University of Michigan, Ann Arbor, Michigan, United States ivylin@umich.edu
}

\begin{abstract}
We report on the design, premiere and public evaluation of a multifaceted audience interface for a complex non-linear musical performance called Climb! which is particularly suited to being experienced more than once. This interface is designed to enable audiences to understand and appreciate the work, and integrates a physical instrument and staging, projected visuals, personal devices and an online archive. A public premiere concert comprising two performances of Climb! revealed how the audience reoriented to the second performance through growing understanding and comparison to the first. Using trajectories as an analytical framework for the audience 'journey' made apparent: how the trajectories of a single performance are embedded within the larger trajectories of a concert and the creative work as a whole; the distinctive demands of understanding and interpretation; and the potential of the archive in enabling appreciation across repeated performances.
\end{abstract}

\section{Author Keywords}

Spectator interfaces; music; performance; journey; trajectory; piano; instrument; public displays; archives.

\section{ACM Classification Keywords}

H.5.m. Information interfaces and presentation (e.g., HCI): Miscellaneous

\section{INTRODUCTION}

From VJs to the giant screens of arena-scale pop concerts, projections of various kinds enhance the audience appreciation of musical performance, showing close-up of views of musicians and enhancing aesthetics through

\footnotetext{
(C) Association for Computing Machinery 2018. This is the author's version of the work. It is posted here for your personal use. Not for redistribution. The definitive version was published in CHI 2018, April 21-26, 2018, Montreal, QC, Canada, https://doi.org/10.1145/3173574.3174142
}

skilfully designed graphics. These 'visuals' are increasingly spilling out onto mobile devices, for example interactive programmes at classical concerts that allow audience members to access programme notes or even to follow progress through the piece in real-time. Musical performances involving digital technologies may demand even greater levels of visual enhancement and explanation due to the complexity and novelty of their forms, where their functions and mechanisms are often hidden from view.

We report on the iterative design of an interface intended to support an audience in understanding and appreciating a complex, non-linear musical work called Climb!. Working closely with the composer, Maria Kallionpää (who is a coauthor on this paper), we have designed and publicly trialled a set of supporting interfaces for the work including projected visuals, a mobile application and an online archive. Any creative work can be re-experienced, often in different ways, for example a live concert may be revisited as a recording. But Climb! is particularly well suited to reexperiencing as it has a variable non-linear structure, such that the arrangement of each performance depends partly on the pianist's actions and partly on chance. In this respect, it is broadly representative of a wider range of interactive experiences from games to museum guides.

From a music perspective, our challenge was to enhance audience appreciation of a complex interactive music performance that involves an element of repeat listening. Our design aims were: to strike an appropriate balance between allowing the audience to appreciate the music in its own right while scaffolding understanding and interpretation for those who wish it; to integrate resources and cues across both projected and personal screens in a way that was sensitive to the artistic intent of the work; and to accommodate diverse patterns of repeated listening, ranging from a single performance, through multiple performances in a single concert to following the work over a life-time of performances.

From a general HCI perspective, our work speaks to the design of spectator interfaces that reveal (or otherwise) interactions with computers to observers [32] and also to 
calls that interfaces should be "open to interpretation" [34]. Specifically, we draw on the concept of trajectories to help reflect on the HCI challenge of designing audience journeys that unfold over complex and repeated performances.

Our contributions are the descriptions of the interfaces, the findings from the premiere performance, and a set of recommendations about designing unfolding audience journeys through repeated interactive user experiences.

\section{Understanding the musical experience}

Our aim in this paper is to scaffold understanding and interpretation of the work rather than to unpick audience members' specific interpretations, which are problematic to unravel given the rich complexity of context, prior experience and social factors [36]. Thus our concern is not with the specifics of music perception and cognition [e.g., 26], emotional or physiological response [e.g., 27], cultural references and associations [e.g., 28, 36] or any lengthy discussions about aesthetics. Rather we take an ecological view and acknowledge audiences form understanding on their own terms and "perception must be understood as a relationship between environmentally available information and the capacities, sensitivities, and interests of a perceiver" [8].

The experience of new or challenging music calls for the development of a new "interpretive platform" requiring supplementary resources [3]. Radborune et al.'s index for measuring the audience experience notes that "Knowledge is concerned with the audience's need for information to enable a better understanding or perspective of the performance with which they are engaging" and this knowledge can be presented through a variety of channels, such as visual enhancements, self-interpretive aids, and preperformance talks [30]. Brown \& Novak's [6] study of intrinsic impacts from live performance observed those less experienced and knowledgeable audiences who attended pre-performance enhancement events reported an increase in readiness and subsequent impact. Furthermore, Radbourne et al. [30] state "audiences see active and connected forms of engagement as indicators of quality and that this influences re-attendance".

John Dewey describes 'an experience', as a self-contained entity that concludes when the "material experienced runs its course to fulfillment" [11]. In contrast Reason [31] argues that an experience of a performance lives on beyond the event through "multi memories, connotations, reflections and afterlives which take place in audiences' social and imaginative lives". This latter view speaks to our work but in addition we consider how repeat performances of the same work can further extend and enhance the lifetime of 'an experience' of a musical work.

\section{METHODOLOGY}

We follow the approach of Performance-Led Research in the Wild [2]. Broadly construed, this is a flavour of Research Through Design [17] in which research findings emerge from reflection on the practice of designing and making artefacts. The "performance-led" element refers to the artefacts that are created being artistic performances. It is important that these are driven by the vision and needs of professional artists, with HCI researchers initially acting as technical facilitators. The "in the wild" element refers to touring the resulting performances to professional venues, with HCI researchers studying how they unfold from both artists' and audiences' perspectives. Reflections on both design and experience typically shape some form of generalized knowledge, often so-called 'intermediate' design knowledge that sits somewhere between grand theory and specific design instances [22]. The approach is evident in the HCI literature since 2001, though was only 'badged' as such in 2015 .

In this specific case, we have worked with a professional composer and pianist to create and perform an interactive work for classical piano called Climb!. In what follows, we report on a nine month process spanning the composer's initial design; staging the premiere performance; and lessons learned from this. We focus in particular on the evolution of a suite of spectator interfaces including a projection interface, a mobile app and an online retrospective archive, that together support repeated engagement with the piece.

\section{THE INITIAL DESIGN OF CLIMB!}

We begin with a concise overview of Climb! so that the reader can appreciate the nature and structure of the work, its variability and also why it is challenging for audiences to understand. We refer the reader to the accompanying video that presents documentation from its first performance.

\section{Musical form and structure}

Stylistically, Climb! [23] is situated at the intersection of a classical romantic virtuoso piece, a contemporary indeterminate work and an interactive game, intended for concert hall performance. Climb! is a pre-composed nonlinear work scored in traditional notation. The score comprises 17 micro-compositions called events that are connected into three macro-compositions called paths with a set of cross-links that branch between them at certain points. The composition is intended to unfold differently in every performance in response to choices or challenges that the performer encounters. The overall theme of the work is of a journey up a mountain. Each event offers a differently themed musical encounter, for example being attacked by a flock of birds, finding a shimmering stone or experiencing an hallucination. Beyond their expression by the pianist, these encounters are characterised in two further ways, first as audio effects representing 'weather' that augment or transform the instrument's sound, and second, where the piano physically duets with the performer, playing its own parts alongside theirs. The latter is possible because the work is written for Disklavier piano that can be controlled using MIDI - physically actuating its own keys - at the same time as being played as a conventional instrument. 


\section{Realisation}

The performer chooses their route and also triggers audio effects and Disklavier parts directly through their playing (rather than pressing buttons, pedals, making gestures or other 'out of band' forms of control). This utilizes a technology called Muzicodes [18, 19] that enables phrases of music to be pre-defined as musical codes or triggers, analogous to the anchors of hyperlinks. The system can be programmed to trigger various actions on detecting a successfully played code including - in this case controlling MAX/MSP audio effect patches, playing MIDI files into the Disklavier, jumping to new locations in the score and triggering onstage projected visuals and updates on a mobile app. The pianist plays from a dynamic digital score [40] which has previously been integrated with Muzicodes [23]. Thus the performer controls a complex array of audio-visual interactions directly by her performance of pre-composed material. Some of the musical codes are deliberately challenging to play, and the pianist's success or otherwise affects her route through the indeterminate arrangement of the composition's events and paths. This raises something of a challenge for audiences to understand what are for the most part hidden interactions. In response to this challenge we introduced three further elements to the work: projected visuals, a mobile app and an online archive, described below.

\section{Interaction design approach}

In discussion with the composer we agreed on a common approach to interaction design for the work. Our goal was to enable the audience to appreciate the piece, first and foremost, as an enjoyable and coherent musical work, at the heart of which is the unfolding interaction between the pianist and the Disklavier/system. As an artist-led project, we were not trying to design a product that would please everyone, but rather were seeking to help the composer to realise her vision for the work. Consequently, each interface was intended to complement the live performance without undermining it or unduly distracting from it. The various interfaces are also intended to complement and be coherent with one another. As with typical traditional programme notes these interfaces seek to provide some cues and resources to inform the listener's interpretation of the work (e.g. the over-arching narrative of climbing a mountain) but without attempting to prescribe a single rigid perspective.

\section{Projected visuals}

We created accompanying projected visuals to augment the experience. Our intention was that these would enhance the mood and atmosphere of the piece while providing some cues as to what was happening, but avoiding overt explanations. As shown in Figure 1 and the accompanying video, they took the form of a series of animations based around the shape of a mountain. The body of the mountain displayed a bespoke animation for each section of the journey; the current weather was overlaid upon it; its sides would shake in synchronisation with the self-playing Disklavier parts; and the background would briefly turn red whenever a Muzicode was triggered. These visual elements are sufficiently synchronised to the music that they may be regarded as 'audiovisual entities', as commended by Correia et al. [9]. These four layers of visual animations were rendered in a browser (using WebGL and HTML5) for onstage projection.

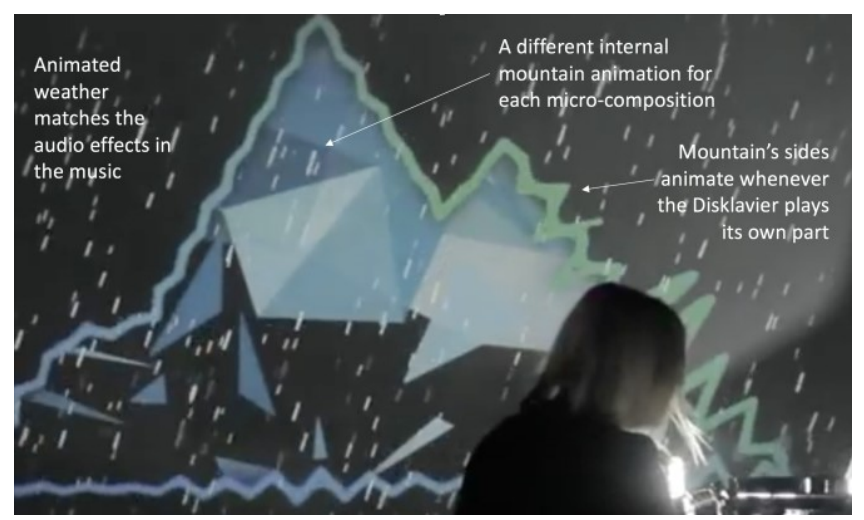

Figure 1 CThe University of Nottingham. The Climb! projected interface

\section{Mobile app}

We also created a mobile audience app to help further explain the work. This gave more explicit (didactic) information. The app synchronized with the performance to the extent that it showed which section of the piece was currently being played. Before the performance the app showed a short programme note. During the performance it showed a dynamic map (Figure 2) of the performer's progress up the mountain, including the current, previous and possible next sections. A short narrative description was also provided for the current section, e.g. "Avatar encounters an angry deer". After the performance the app showed the complete path taken and text narrative for that performance.

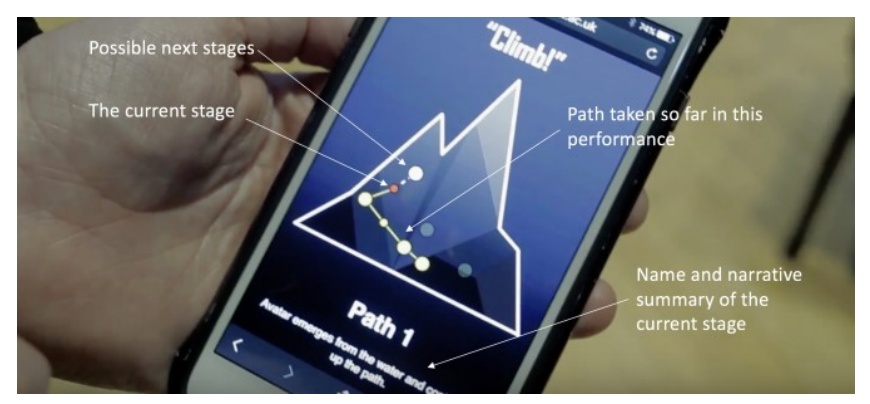

Figure 2 (The University of Nottingham. The Climb! mobile app

The Climb! archive

Finally, immediately after the premiere we created an interactive archive to enable audiences (and others such as scholars and researchers) to review and compare different performances of Climb!. Thus an audience member who only saw one live performance could still compare their experience to other performances. Figure 3 shows the archive interface. Selecting a given performance visualizes its route onto the mountain view. Entire paths or single 
events can be selected and played as audio or video recordings. Playback is augmented with text notes on which sections are being played and when given Muzicodes were triggered. The archive video for the dress rehearsal is a single continuous shot of the stage. The other archive videos for the premiere are edits interleaving material from the stage overview, a close-up of the performer and a closeup of the keyboard.

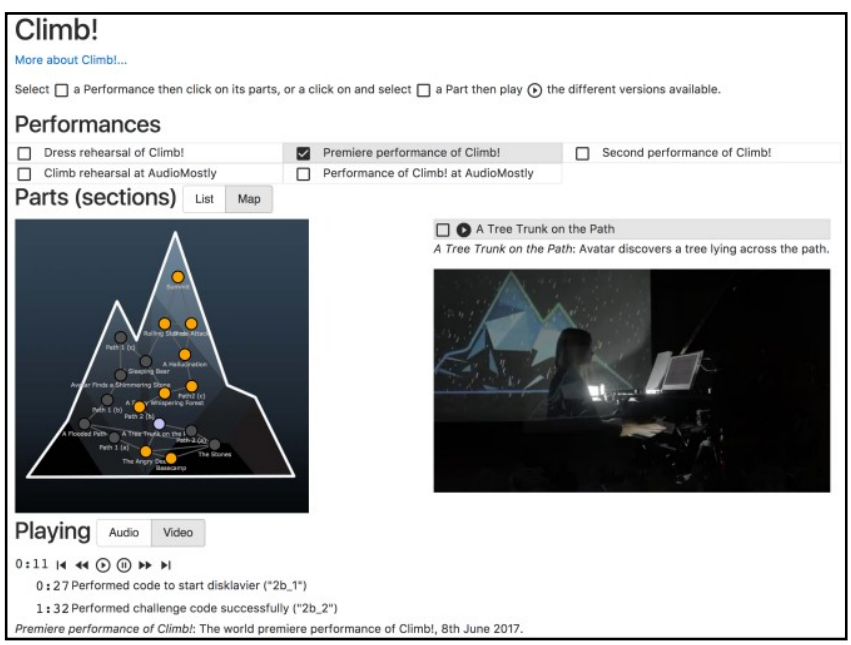

Figure 3. The Climb! archive interface

Our long-term aim is that Climb! will become a selfdocumenting work in which every performance (and perhaps every rehearsal) by any performer anywhere will be captured and logged in the central archive as it tours.

\section{THE PREMIERE OF CLIMB!}

The premiere of Climb! took place in a classical recital hall as part of a regular series of contemporary music recitals at the University of Nottingham. The composer performed the work herself. The audience was a mixture of regular attendees for the event and those who had been invited by the research team. The regular attendees had a specific interest in contemporary music. Other attendees had much more diverse (mainstream) musical preferences, but most attendees reported at least "good" expertise in music.

The recital was structured as two performances of the work, each lasting just under twenty minutes (with a short presentation between), with the idea that the audience would be able to hear two contrasting versions. While it is unusual for a work to be performed twice in the same programme this allowed us to directly address the issue of repeat performances of a dynamic and non-linear work from the audience's perspective. We chose to give the audience minimal explanation of the work before the first performance, with only a high level description of the piece in a paper and app programme note. This allowed the audience to gain a first impression of the music and visuals 'in their own right' before then learning about the concept, structure and technology. We then gave them a verbal explanation of its structure and realisation before the second performance, together with more detailed programme notes.
The paper programme note given to all audience members included a link and QR code for accessing the web-based app, which worked on most smart phones. Smart phones were also lent to audience members who had trouble running the app at the start of the concert.

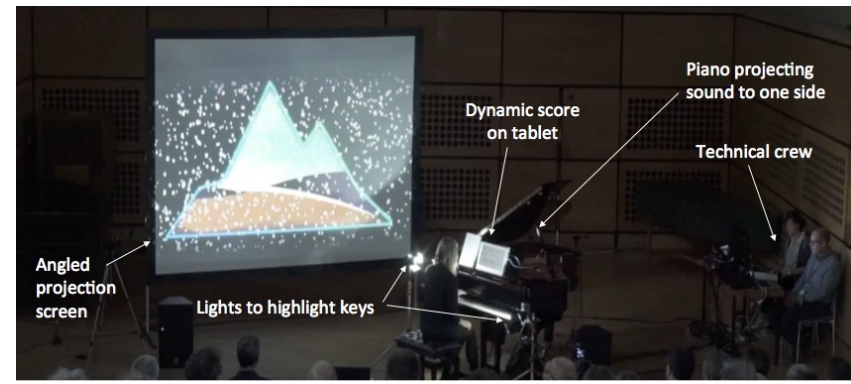

Figure 4 (C) The University of Nottingham. The staging of Climb!

Figure 4 shows the physical organisation of the stage area. Conventionally, the piano would be aligned with the open lid facing the audience. In this case however, the piano was turned approximately $45^{\circ}$, making the performer more visible but at the cost of projecting its sound off to one side. We also chose to light the piano keys closely from two sides at oblique angles so as to emphasise their movement (through shadows). We placed the projection screen at an angle behind the instrument, and set the computer and mixing desk (and their operators) off to one side so that they were not directly part of the performance space while maintaining line of sight with the pianist in case of any technical difficulties.

\section{AUDIENCE FEEDBACK FROM THE PREMIERE}

To gather feedback from the audience we used a paper questionnaire that captured basic demographic details and various questions about their experience of the performance and suggestions for improvements. One version of the questionnaire was completed after the first performance and a second after the second performance. With their explicit consent we also interviewed some members of the audience to video camera after the show in order to capture more detailed thoughts and reactions. The team then met with the artist several times to discuss the data and determine further developments to the work. Results were analysed using the nparLD [24] package for nonparametric analysis. Significance results are nparLD's non-parametric ANOVAType Statistics (ATS). These generalise the more commonly used Kruskal-Wallis (between subjects) and Wilcoxon signed ranks (within subject) non-parametric tests to multiple variables, while an ANOVA cannot be used because the individual questionnaire responses are ordinal values. Comparisons include all participants completing that item for both performances.

Questionnaires for both performances were completed by 39 audience members (around 50 people attending the performance in total). 21 were female and 18 male. 15 were aged below $30 ; 13$ between 30 and 50; 8 between 50 and 
65 ; and 3 were over 65 . They claimed a high level of expertise in music (median of 4 (good) on a 5 point Likert scale between very poor and very good) and slightly less expertise in technology (median of 3.5).

\section{Audience enjoyment of the performance}

We asked audience members to rate whether they "enjoyed the performance" on a seven point Likert scale (from 1, strongly disagree, to 7 , strongly agree). The median score after each performance (i.e., at the interval and at the end) was 6 (agree), with no significant change but with a slightly wider spread of opinions after the second performance, with some liking it more and others liking it less (see figure 5).

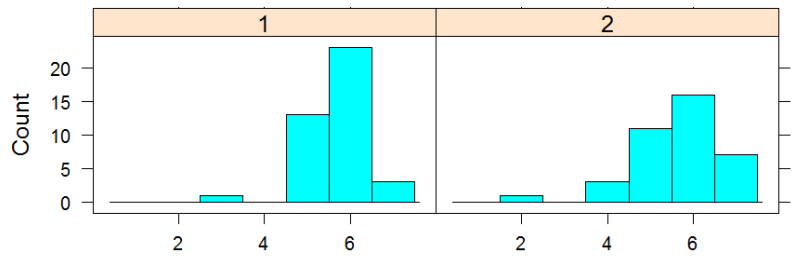

Figure 5. Responses to "I enjoyed the performance" for the two performances of Climb!

We then asked what they did and did not like about the performance, considering their responses in the light of the audience Q\&A and interviews. Some commented favourably on the music: "Very powerful, atmospheric music, loved the piano sonorities. Painted a cold vivid picture of adventure". However, a few were critical of the narrative: "I don't think the narrative was anywhere near as sophisticated/interesting as the music". Some appreciated the melding of music and technology, including the game like elements: "My son who is only 7 was captivated. $\mathrm{He}$ would have not sat through a typical piano concert this long. He liked the game elements most". But others were more critical of the general concept: "I didn't really get it. Stories have been told with music for centuries. What does a smartphone add that a PowerPoint slide can't?". Such divergences of opinion are common with artworks, and indeed part and parcel of making personal interpretations.

\section{Audience understanding of the performance}

We also asked audience members to rate whether they "understood the performance" on the same 7 point Likert scale. Understanding was mixed for the first performance (median 5, somewhat agree) and significantly higher after the second performance (median 6, agree; $\mathrm{N}=35$, RTE +0.31 , ATS 45.10, 1df, $\mathrm{p}=1.87 \times 10^{-11}$ ) (Figure 6).

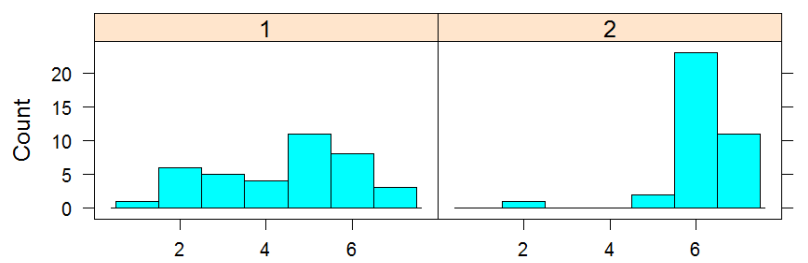

Figure 6. Responses to "I understood the performance" for the two performances of Climb!
This was clearly in large part due to the explanation that was given at the interval as commented upon by many respondents. Their comments also made it clear that for many, such explanations aided their enjoyment as they were then better able to predict and follow the pianist's journey through the work, or experience the various parts as a whole (see specific comments in following subsections).

\section{Interaction with the Disklavier}

Experiencing the battle between the pianist and Disklavier was an engaging aspect of the work: [the respondent enjoyed] "Seeing the disklav move and her perform with it" However, in spite of our efforts to arrange the stage and lighting, seeing this clearly remained a problem, leading some to suggest technical solutions: "the keyboard was visually obscured - could have been projected". People reported attending less to the piano keys in the second performance compared to the first $(\mathrm{N}=37$, RTE -0.198 , ATS $=10.87, p=0.001$ ), perhaps because of its initial novelty. In at least some cases the change between performances was towards more specific and focused attention: "The 1st made you listen to $\&$ watch the pianist $\&$ piano. The 2 nd made you try to spot the key trigger."

\section{Projected visuals}

The projected visuals were generally well received as being as essential part of the work, for example: "the use of musical expression along with the visuals of both the projection and the performance, conveyed the intended scene very well". However, some did not find them beneficial: "I did not like the graphic. I found it distracting" while others wanted to see video projection of the pianist's hands (see above) or "a journey represented on the screen" (as in the mobile app). There was no significant change in the amount that people reported attending to the projection in the second performance, although some people's appreciation of it was influenced by explanation in the interval: "Enjoyed video more on second when understood the cues (which didn't pick up on at first)."

\section{The mobile app}

From questionnaires 34/41 (83\%) people used the app during the first performance and 29/39 (74\%) during the second. The app was seen as useful and an enhancement as far as it went, although there were many suggestions for how it might be extended. Some comments revealed how the app made visible what was happening but not why: "the app made it understandable, I followed the journey but had no clue how path was chosen etc.". Some (presumably with a high level of musical knowledge) wanted to see the score: "the app gave me information and I could see some of what was happening, but would have liked to see what the pianist was seeing". While clearly useful to some, the app was a distraction for others, both generally but also because it inevitably brought the outside world into the performance setting: "mobile app made other things too available. For example my email that gives me notices, and the possibility to google related stuff'. Finally, the audience discussion and interviews suggested that deploying an app in this way 
led people to expect Climb! to be more interactive, such as allowing the audience to vote for the pianist's route up the mountain. There was a small but significant reduction in app use between the first and second performances $(\mathrm{N}=39$, RTE -0.047 , ATS $=4.899, \mathrm{df}=1, \mathrm{p}=0.027$ ).

\section{Comparing performances}

One notably enjoyable aspect of the second performance was to compare the paths between the two performances: "Enjoyed looking for variations" and "interested to see if there were any changes". However, this was perhaps also the biggest cause of disappointment on the evening. While the pianist had intended to perform a quite different route through the second performance, she ended up accidentally playing a very similar one and once committed along this path could not find an opportunity to remove herself from it, in part due to a lack of cross-paths higher up the route. The audience clearly picked up on this: "I expected a different path after the first performance, but most of the content is the same", "I was disappointed that the performance mostly followed the same path (\#2) except for the very beginning" and "not sure how much control she had whether she meant to go up path 2 again".

\section{Changing approaches to the performance}

From the questionnaire comments it is clear that many people engaged with the second performance differently to the first performance, and not only because they now had a previous performance to compare it with (above). In some cases their re-orientation was due to their increased understanding of specific elements, e.g. the projected visual cues or the musical triggers mentioned above. But in other cases they changed strategies for engaging with the performance simply in order to adopt a fresh perspective on the piece, e.g. "I dipped in/out of the different threads more now, which made it interesting in a new way", and [the second performance was] "Different as I ignored the projected video made sense musically without".

\section{Future interest}

Finally, we asked audience members about their future interests in relation to the piece, specifically whether they would like to: find out more about it; go to another performance; have a recording of another performance; have a recording of that specific performance; or have some other unspecified souvenir of the performance. Figure 7 shows the relative effects for each performance; Relative Treatment Effect (RTE) is a normalised (0-1) measure of differences between conditions. Initially the audience most wanted to find out more (median response 6). Interest in going to another performance or a recording of another performance remained stronger than interest in a recording of that performance or another (unspecified) souvenir.

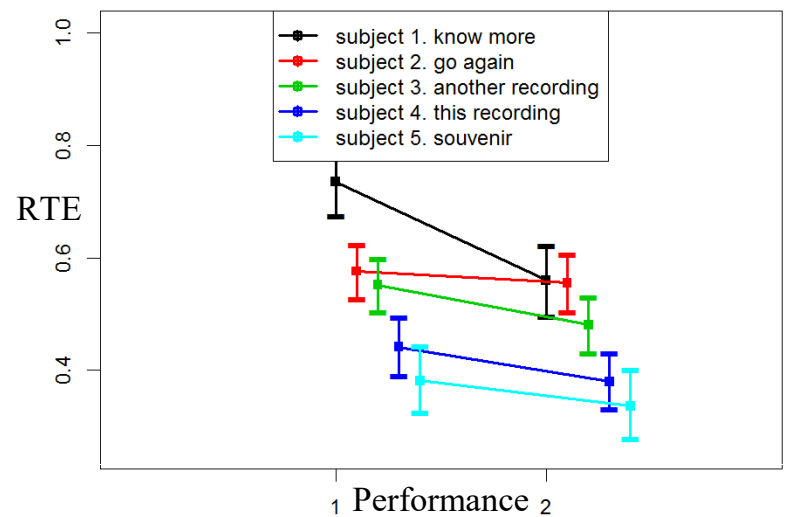

Figure 7. Responses to "I would like..." for the two performances of Climb!

Feedback from the archive

Several weeks after the premiere, when the archive was available, we invited audience members (for whom we had contact details) to explore the archive in their own time. We did not observe their use of the archive but followed up with semi-structured interviews with three audience members as a preliminary evaluation of the archive. At this point the archive was populated with three performances, namely the dress rehearsal and both premiere performances. The continuity of design and content between the live performance and the archive was acknowledged: "Well, seeing the video which was almost from my perspective in the auditorium. It was very evocative on that front, and then having the mountain map on the left-hand side [i.e. of the archive] was reminiscent of the view I had on my phone in the concert. So, having all the different components made it easy to remember in more detail".

Respondents were keen to view and compare the performance of the Dress Rehearsal to their recalled experience of the premiere performances. They appreciated the 'enhanced' perspective that the archive offered, such as the text annotations identifying the location of the codes in the music and the close up of the piano keys, and score view presented within the composited performance videos. They explored the archive actively, viewing and comparing events across different performances, seeking out specific interactions such as the performance of codes. One participant suggested it would be helpful to be able to be able to choose different camera views to focus in on specific facets or interactions in the performance. Participant 2 stated that the archive encouraged an 'investigative' behavior, but also, "it's something that is being re-lived because I can remember what happened. Now if I hadn't seen it, there may be more of a temptation to see it through, but actually it was a kind of a recap and would it allow me to go to certain points that I did find interesting or not." 


\section{JOURNEYS THROUGH AUDIENCE EXPERIENCE}

We now consider and discuss our observations and make a series of recommendations for the design of spectator interfaces to support unfolding audience journeys.

A key concern that runs throughout Climb! is how to enable the audience - who are after all intended to be the primary beneficiaries of the experience - to arrive at an understanding and appreciation of what is going on. Specifically, how might we design audience journeys so as to enable understanding, interpretation and appreciation to unfold over complex and repeated performances? The idea of the user experience being a journey is of course familiar to HCI and is also widely applied across the UX industry. Previous HCI research has argued that the spectator experience - that is the experience of those who observe interactions rather than directly undertake them - also involves a journey, for example from being an unwitting bystander, to being a witting audience member who appreciates what is happening [35].

In what follows, we build on this idea of the spectator or audience journey. We also turn to the 'trajectories' conceptual framework to provide an overarching organization for our discussion. The idea behind trajectories is that a user experience - an audience journey in our case can be expressed as the interleaving of three different types of trajectory: canonical (the intended or scripted journey through the experience), participant (the actual journey as experienced by a participant) and historical (the subsequently recounted experience) [38]. We consider each type of trajectory in turn, using it as a lens through which to inspect the various challenges of and approaches to designing audience journeys. In this case, we apply trajectories to the audience journey rather than the pianist's (who is the primary interactor with the technology).

\section{Canonical: staging interpretation}

Canonical trajectories are pre-scripted and shape the experience from the outset. They are the plan. But how easy should it be to interpret a given cultural work in the first place, and how much understanding should an audience have? This is largely a matter of artistic or perhaps curatorial choice. Moreover, it may be quite possible to readily understand how an artwork is made, while its meanings (the why of the matter) may elude simple interpretation. As Sengers and Gaver note from an HCI perspective, "designs can clearly specify usability, while leaving interpretation of use open" [34]. In Climb! we arrived at the position of wanting to assist the audience in clearly understanding the form of the work and the mechanics of its delivery while leaving the musical interpretation of the work more open.

\section{Making performance visible}

To this end, Climb! adopts what Reeves et al. [32] term an expressive strategy, aspiring to reveal both the performer's manipulations of the interface and their consequent effects to the audience. However, realising this is a particular challenge for interactive musical performances in that, as Berthaut et al. [4] note, audiences often lack information about the instruments being played and complex arrangements of hardware and hidden software mappings can render it difficult to see musician's gestures or relate them to musical effects. Climb! combines several tactics to make the work of performance visible including reorienting the piano to face the audience and using the projection to make visible cues to otherwise hidden elements of the system (as noted by Dannenberg [10]). There was also considerable stagecraft involved in juxtaposing and aligning the various interfaces and elements and in carefully lighting them so that they could be seen by the audience in appropriate relationships to one another, from different vantage points in the theatre, all without overly compromising sound quality. However some audience members still struggled to see clearly the Disklavier's interaction with the pianist, and in future performances we intend to integrate live video of the keys and the pianist's hands into the projected view (similar to [4]).

Recommendation: decide a strategy for revealing the core act of performance to the audience that is consistent with the artistic intent (e.g. expressive), tailor interfaces to support this, and arrange these carefully on the stage.

\section{Cues and resources for interpretation}

The artist may also provide a further diverse set of specific cues to help the audience interpret the performance, bearing on the why of the work, not just the how. For example, Vines et al. note how video DJ's physical movements and facial expressions of performers convey information about the emotion of music [39]. In Climb! the projected visuals include 'narrative' cues that relate to the meaning of the work, e.g., weather animations. Similarly, the mobile app provides cues to the current section and narrative. In addition, as is common place at many concerts, the producers who organized and hosted each performance produced a set of programme notes (that provided the audience with an initial framing of the work, summarising its inspiration, form and use of technology), demonstrating how this task is shared among various stakeholders including: the original composer/artist; the current performer and their crew; and also the producers. However the details of the musical challenges remained obscure to most audience members. So in future performances we intend to provide additional cues (in the projection and the app) about impending challenges.

Recommendation: consider a variety of additional interpretive cues (to the why of the work as well as how) to complement the experience as a whole.

\section{Unfolding experiences}

Looking beyond the moment of performance, what information should the audience receive before, during and after a particular performance? Several researchers have argued for designing journeys through experiences that unfold over time. We also reflect back to Reason's view of 
'an experience' as being as continuing process [31]. In discussing the interactive VR artwork Traces, Penny et al. describe an "autopedagogic" approach in which the complexity of mappings and representations increased over time so as to help the viewers' understanding gradually unfold [29].

Given the repeated structure of the premiere, our initial strategy for Climb! was for the first encounter with the work to be relatively open, unscaffolded and potentially ambiguous. Our experience has led us to modify our strategy for future performances, because, at least for some audience members, the additional understanding provided before the second performance was reported to enhance their enjoyment. At first sight this contradicts Bin et al.'s [5] findings that a pre-concert technical introduction to a digital musical instrument did not increase self-reported enjoyment or interest. However they are dealing with finegrained interaction with unfamiliar instruments, and they acknowledge that their specifically technical introduction may not have increased the 'transparency' of the performance. We are dealing a situation more analogous to a duet where one of the performers is not immediately obvious, and so a simple explanation may have more direct impact on enjoyment. Therefore in future performances the app will provide access, for those who want it, to diverse resources for understanding and interpretation (e.g. how the system works) even before the performance starts.

Recommendation: consider when resources for understanding and interpretation should be made available, specifically before, during and after a given performance.

Trajectories through repeated experiences

Each individual performance of Climb! has its own particular sequence of musical paths and events. Thus, considering a single performance, the canonical trajectory of Climb! is intentionally both variable and to some extent unpredictable (e.g., will the performer successfully play each code and what will the weather be?). This increases the expected differences between performances of Climb!, and appears to contribute to the audience's sustained interest in future performances and different recordings.

However, Fosh et al. [15] draw attention to the multi-level character of trajectories, including the presence of both "local" and "global" trajectories even within a single experience. Considering Climb! as a work, incorporating all of its performances, recordings and other manifestations (e.g. score, archive) reminds us that an individual's trajectory of engagement with the work may span multiple performances spread across multiple events, and may also include other points of engagement in between, for example with the archive, with recordings or with a published score. Future performances may also deliberately steer the work in new directions, for example to contrast different pianists' performances and experiences of the work.
Recommendation: the trajectory for each individual performance should be designed to fit within an overall lifetime trajectory of engagement with the experience.

\section{Participant: enabling personalised journeys}

Participant trajectories express how individuals may adapt the canonical trajectory for themselves as an experience unfolds. They are the situated action [37]. People came to our performances with diverse motivations, interests, and knowledge. Some were interested in the narrative of the work, others focused on its musical structure, some turned to the technical aspects, while others wanted to relax and enjoy the experience. Moreover, some had previously engaged in rehearsals and demos, while many had not. Consequently, engagement with an experience like Climb! is very much a personal matter.

\section{Convergence and divergence}

This can be conceptualised as the divergence and convergence of participant trajectories. There may be critical times and aspects of the piece where convergence is desirable between participant and canonical trajectories, perhaps at the beginning or the climax of the piece when the performer might want to focus the audience's attention as a whole. Similarly, some convergence between different participants' trajectories may be desirable, e.g., for every audience member to have some shared orientation to the piece at the end of a performance. But this needs to be balanced with the ability for audiences to diverge from the canonical trajectory to some extent, for example to selfselect the cues that are most relevant to them in the moment. This tension or flux between convergence and divergence of participant trajectories requires careful design and negotiation.

Recommendation: design and support key elements of convergence within the performance, for example with additional shared cues, but allow space for individual interests and perspectives.

\section{Context and Comparison}

Audience members enjoyed being able to compare the second performance with the first. This allowed them to relate their current experience to past experiences (especially their own), and to change their listening strategy accordingly, e.g., attending to specific details of musical events they have heard before. This can be readily generalised to include appreciating the current performance of a piece of music (or other work) in the wider context of previous ones.

Recommendation: allow audience members to compare their current experience with past experiences, especially their own.

\section{Public and personal screens}

There is already a substantial literature in HCI about combining public and personal displays. For example secondary personal screens are normally used for peripheral awareness of content and as a companion of the primary 
display [20]. [25] argue for using large public displays to show complex multi-media presentations and secondary screens for special annotations according to individual preference. Considering multiple-screens in relation to watching television four main purposes of secondary screens have been suggested: to control, enrich, share and transfer content [7].

While broadly reflecting these strategies there are nuances that are worth highlighting in Climb!. Considering first the projected video, unlike many multiscreen situations (e.g., with television) the projection itself is secondary to the piano and pianist, and is under the exclusive control of the artist. The public display is therefore a good location for cues that augment what is visibly happening nearby on stage right now (e.g., additional video views of the performer). It also suits material that the artist deems to be an integral - canonical - part of the work, for example more impressionistic animations that enhance its aesthetic and that may themselves require interpretation. On the other hand, our use of personal displays more closely mirrors common patterns of use, whereby additional supportive information can be tailored to individual interests without affecting others. In our case, the diverse cues and resources that are offered through the mobile app are intended to support divergence between participant trajectories while the projected view encourages convergence.

Recommendation: divide cues and resources between public screens, prioritizing artist-led and canonical material; and personal screens, prioritizing individual and supporting information - so as to balance divergence and convergence.

\section{Orchestration}

In the trajectories framework, orchestration refers to the shaping of an experience from behind the scenes, for example by technicians using monitoring and control interfaces. In Climb! the technical operator of the system could intervene to some extent to shape the performance, for example manually marking codes as triggered successfully. When in the second performance of Climb! the pianist found that she was unable to get the system to follow her intended path the technical operator might have responded by nudging her along a particular path anyway (though in this case did not). Whether and how to reveal such orchestration work is another key choice that affects audience appreciation of the work. It is common in many digital musical performances for the 'technicians' who operate laptops and so forth to appear on stage alongside the main performer, echoing a general aesthetic that celebrates the presence of digital technologies. On the other hand, hiding orchestration work may afford greater opportunities for dealing with technical difficulties without distracting from the main focus of the experience - the approach adopted in Climb!

Recommendation: consider whether or not to reveal orchestration work and if so, how to provide additional cues and resources to help audiences appreciate this.
Historic: archiving for repeated experiences

Finally, historic trajectories capture how participants reflect on and talk about an experience afterwards. A good illustrative example from beyond musical performance is the Automics mobile app [12] that enabled visitors to theme parks to create personal stories of rollercoaster rides by combining their own photos with automatically captured ones using comic-strip templates. This notion of the historic trajectory captures the importance of reflecting on experiences which connects to our interest in designing for repeat performances of Climb!.

\section{Integrating the archive}

For Climb! the archive has the potential to be a central resource to support audience understanding. Evaluation of the archive is necessarily preliminary given its limited use to date. However our goal is to make Climb! a richlyarchived work, by which we mean making the archive a 'first class' part of the experience itself, directly exposing it to the audience in order to support their continuing experience. For example, in future, audiences will be able to visit the archive after a performance to compare 'their' show with others, or use the mobile app to compare performances during a show. So even a concert with a single performance can be compared to others, responding to the audience's sustained interest in going to future performances and hearing recordings of different performances. The archive was also deliberately designed to include much of the visual content and 'look' of the staged work and this appeared to support users in bridging their live and online experiences. The Climb! archive was described as an interactive resource that "compliments", enables reflection on and subsequent investigation of multiple live performances. The archive grants control to the user and is coupled with additional performance information, or perspectives, not available on-stage (e.g. the performer's view of the score, annotations). This in turn can enhance a user's understanding and extend their experience (in the sense of [31]).

Recommendation: consider integrating the live experience with a complementary and consistent archive of past experiences.

\section{Extended sequences of performances}

To date there have only been two public performances of Climb!, and these were both within a single concert event. One must ask, therefore, what effect it would have if audience members were to attend performances over a much longer timescale, and/or attend more than two performances of the work. Addressing these questions empirically must wait for future performances. However we can extrapolate to some extent from other works and domains. Repeat experience is common across many cultural forms, even conventional linear ones. The same story may be retold and reinterpreted many times over the years, often across different media. And some cultural events, for example festivals, are also cyclic and repeated, opening up possibilities for comparison to previous years. 
We saw with Climb! that the expressed interest in going to another performance remained strong even after the second performance, while the expressed interest in hearing a recording of another performance remained quite strong (and stronger than the interest in hearing a recording of the same performance).

As future performances become more separated in time we might anticipate that audience members will recall the earlier performance(s) less well. Mirroring a regular concert audiences' responses to popular works, some listeners might wish then to revisit previous performance(s) as a kind of preparation. Others, however, may well prefer to hear the work afresh. Similarly, as an individual experiences more performances of the work they may choose to listen 'anew' on each occasion, although presumably with some growing sense of familiarity and anticipation, at least in the repeated fragments. On the other hand, some listeners may choose to 'invest' in the piece, developing their own knowledge and expertise, for example through explicit review and comparison of past performances.

Our experience of Climb! also sensitises us to the historic trajectory as being an essential element of designing audience journeys through extended user experiences. The historic trajectory is about reflection and storytelling and so inherently involves the act of making and sharing an interpretation as part of an unfolding journey. The archive opens up possibilities to publish different recorded versions of the work. Indeed, the Climb! archive is already a nonlinear form of recording, for example a user can compare all of the performances of a particular micro-composition. However, it might also be used to generate more conventional linear formats such as playlists, CDs, DVDs or perhaps even an 'as-live' playback though a Disklavier piano. Finally, the archive can support performers in planning further performances - feeding back into the canonical trajectory - by enabling them to choose interesting routes as well as learn from previous players.

\section{CONCLUSION AND FUTURE WORK}

Technically complex and non-linear interactive performances place particular demands on audiences in terms of appreciating what is going on and ultimately in arriving at some kind of personal interpretation. Our experience from Climb! reveals how supporting audience understanding is a multifaceted challenge that touches upon several aspects of interaction design.

The key insight to emerge from our work is that experience designers (composers, performers and technicians) need to carefully design audience journeys - or trajectories - to shape an unfolding understanding of a work. This is true both within a given performance (considering what the audience experiences before, during and after the show) but also across many performances over the lifetime of the work (so that audiences can compare different performances, for example). Further complexities arise from the personal nature of interpretation which leads audience members to require various and different cues to scaffold their individual understandings, with these cues being spread across multiple interfaces, both projected and personal. We also note the potential of archives for capturing and making available supporting materials over the lifetime of a work, with archives being designed for audiences and with works perhaps becoming self-archiving.

Finally, we highlight three areas for future work. First, we have identified several areas in which the current interfaces can be improved, including incorporating live video into the projection, adding more cues for challenges and more interpretative resources in the app. We aim to refine the current interfaces and to evaluate these refinements in a further round of performances. This will also provide the opportunity to begin to explore the impact of longer sequences of performances. Second, although beyond the current scope of Climb!, personal screens clearly have the potential to support audience interaction with works of this kind (and may even lead people to expect it). Previous HCI research has explored approaches such as cheering and applauding [1], voting [21], participating in real-time generation of the score [16], or suggesting moods that prompt the performers to improvise [13]. It would be valuable to address such interaction more explicitly within the audience journey. Third, the current archive doesn't yet support the kind of personalised storytelling that was reported in [12], e.g., the combination of official documentation with people's own media using templates. This might also extend to allowing people to weave their own photos, videos and comments into the archive, similar to examples of crowd sourcing rich media associated with music concerts [33] and marathon races [15]. And specialist versions of the archive might integrate and augment the digital score as a key facet of the experience.

\section{ACKNOWLEDGMENTS}

This work was supported by the UK Engineering and Physical Sciences Research Council [grant numbers EP/L019981/1, EP/M000877/1], the Kone Foundation and the University of Nottingham's Research Priority Area funding scheme.

\section{DATA ACCESS STATEMENT}

Anonymous questionnaire data underlying this paper can be obtained from http://dx.doi.org/10.17639/nott.341 The Climb! performance archive is accessible as http://music-mrl.nott.ac.uk/1/archive/explore/Climb (8th Jan. 2018).

\section{REFERENCES}

1. Louise Barkhuus and Tobias Jørgensen. 2008. Engaging the crowd: studies of audience-performer interaction. In CHI '08 Extended Abstracts on Human Factors in Computing Systems (CHI EA '08). ACM, New York, NY, USA, 2925-2930. DOI: https://doi.org/10.1145/1358628.1358785

2. Steve Benford, Chris Greenhalgh, Andy Crabtree, Martin Flintham, Brendan Walker, Joe Marshall, 
Boriana Koleva, Stefan Rennick Egglestone, Gabriella Giannachi, Matt Adams, Nick Tandavanitj, and Ju Row Farr. 2013. Performance-Led Research in the Wild. ACM Trans. Comput.-Hum. Interact. 20, 3, Article 14 (July 2013).

DOI=http://dx.doi.org/10.1145/2491500.2491502

3. Dawn Bennett and Jane Ginsborg. 2017. Audience Reactions to the Program Notes of Unfamiliar Music. Psychology of Music. https://doi.org/10.1177/0305735617721339

4. Florent Berthaut, Mark T. Marshall, Sriram Subramanian and Martin Hachet. 2013. Rouages: Revealing the mechanisms of digital musical instruments to the audience. In New Interfaces for Musical Expression, May 2013, Daejeon, South Korea.

5. S. Astrid Bin, Nick Bryan-Kinns, and Andrew P. McPherson. 2016. Skip the Pre-Concert Demo: How Technical Familiarity and Musical Style Affect Audience Response. In NIME'16, July 11-15, 2016, Griffith University, Brisbane, Australia.

6. Alan S. Brown and Jennifer L. Novak. 2007. Assessing the intrinsic impacts of a live performance. San Francisco, CA: WolfBrown.

7. Pablo Cesar, Dick C.A. Bulterman and A.J. Jansen. 2008. Usages of the secondary screen in an interactive television environment: Control, enrich, share, and transfer television content. In European Conference on Interactive Television (pp. 168-177). Springer, Berlin, Heidelberg. DOI: https://doi.org/10.1007/978-3-54069478-6_22

8. Eric Clarke. 2005. Ways of listening: An ecological approach to the perception of musical meaning. Oxford University Press.

9. Nuno N. Correia, Deborah Castro, and Atau Tanaka. 2017. The Role of Live Visuals in Audience Understanding of Electronic Music Performances. In Proceedings of the 12th International Audio Mostly Conference on Augmented and Participatory Sound and Music Experiences (AM '17). ACM, New York, NY, USA, Article 29, 8 pages. DOI: https://doi.org/10.1145/3123514.3123555

10. Roger B. Dannenberg. 2005. Interactive visual music: a personal perspective. Computer Music Journal, 29(4), 25-35.

11. John Dewey. 2005. Art as Experience. Penguin.

12. Abigail Durrant, Duncan Rowland, David S. Kirk, Steve Benford, Joel E. Fischer, and Derek McAuley. 2011. Automics: souvenir generating photoware for theme parks. In Proceedings of the SIGCHI Conference on Human Factors in Computing Systems (CHI '11). ACM, New York, NY, USA, 1767-1776. DOI: https://doi.org/10.1145/1978942.1979199
13. Gyorgy Fazekas, Mathieu Barthet and Mark B. Sandler. 2013.. Mood conductor: emotion-driven interactive music performance. In Affective Computing and Intelligent Interaction (ACII), 2013 Humaine Association Conference on (pp. 726-726). IEEE. DOI: https://doi.org/10.1109/acii.2013.165

14. Martin D. Flintham, Raphael Velt, Max L. Wilson, Edward J. Anstead, Steve Benford, Anthony Brown, Timothy Pearce, Dominic Price, and James Sprinks. 2015. Run Spot Run: Capturing and Tagging Footage of a Race by Crowds of Spectators. In Proceedings of the 33rd Annual ACM Conference on Human Factors in Computing Systems (CHI '15). ACM, New York, NY, USA, 747-756. DOI: https://doi.org/10.1145/2702123.2702463

15. Lesley Fosh, Steve Benford, Stuart Reeves, Boriana Koleva, and Patrick Brundell. 2013. see me, feel me, touch me, hear me: trajectories and interpretation in a sculpture garden. In Proceedings of the SIGCHI Conference on Human Factors in Computing Systems (CHI '13). ACM, New York, NY, USA, 149-158. DOI: https://doi.org/10.1145/2470654.2470675

16. Jason Freeman. 2008. Extreme sight-reading, mediated expression, and audience participation: Real-time music notation in live performance. Computer Music Journal, 32(3), pp.25-41. DOI: https://doi.org/10.1162/comj.2008.32.3.25

17. Danny Godin and Mithra Zahedi. 2014. Aspects of research through design: a literature review. Proceedings of DRS, 281.

18. Chris Greenhalgh, Steve Benford, and Adrian Hazzard. 2016. ^muzicode\$: Composing and Performing Musical Codes. In Proceedings of the Audio Mostly 2016 (AM '16). ACM, New York, NY, USA, 47-54. DOI: https://doi.org/10.1145/2986416.2986444

19. Chris Greenhalgh, Steve Benford, Adrian Hazzard, and Alan Chamberlain. 2017. Playing Fast and Loose with Music Recognition. In Proceedings of the 2017 CHI Conference on Human Factors in Computing Systems (CHI '17). ACM, New York, NY, USA, 4302-4313. DOI: https://doi.org/10.1145/3025453.3025900

20. Jonathan Grudin. 2001. Partitioning digital worlds: focal and peripheral awareness in multiple monitor use. In Proceedings of the SIGCHI Conference on Human Factors in Computing Systems (CHI '01). ACM, New York, NY, USA, 458-465. DOI=http://dx.doi.org/10.1145/365024.365312

21. Kate Hayes, Mathieu Barthet, Yongmeng Wu, Leshao Zhang, and Nick Bryan-Kinns. 2016. A Participatory Live Music Performance with the Open Symphony System. In Proceedings of the 2016 CHI Conference Extended Abstracts on Human Factors in Computing Systems (CHI EA '16). ACM, New York, NY, USA, 
313-316. DOI:

https://doi.org/10.1145/2851581.2889471

22. Kristina Höök and Jonas Löwgren. 2012. Strong concepts: Intermediate-level knowledge in interaction design research. ACM Trans. Comput.-Hum. Interact. 19, 3, Article 23 (October 2012), 18 pages. DOI: https://doi.org/10.1145/2362364.2362371

23. Kallionpää, Maria and Greenhalgh, Chris and Hazzard, Adrian and Weigl, David M. and Page, Kevin R. and Benford, Steve (2017) Composing and realising a game-like performance for disklavier and electronics. In: New Interfaces for Musical Expression (NIME'17), 15-18 May 2017, Copenhagen, Denmark..

24. Kimihiro Noguchi, Yulia R. Gel, Edgar Brunner and Frank Konietschke. 2012. nparLD: an R software package for the nonparametric analysis of longitudinal data in factorial experiments. Journal of Statistical Software, 50(12), 1-23. 2012 DOI: https://doi.org/10.18637/jss.v050.I12

25. Michael Kruppa and Antonio Krüger. 2003. Concepts for a combined use of Personal Digital Assistants and large remote displays. Proceedings of SimVis 2003, Pages 349-361, Magdeburg

26. Fred Lerdahl and Ray Jackendoff. 1985. A Generative Theory of Tonal Music. MIT press.

27. Lars Olov Lundqvist, Fredrik Carlsson, Per Hilmersson and Patrik N. Juslin. 2009. Emotional responses to music: experience, expression, and physiology. Psychology of music, 37(1), pp.61-90. DOI: https://doi.org/10.1177/0305735607086048

28. Leonard B. Meyer.1956. Emotion and Meaning in Music. University of Chicago Press .

29. Simon Penny, Jeffrey Smith, Phoebe Sengers, André Bernhardt and Jamieson Schulte. 2001. Traces: Embodied Immersive Interaction with SemiAutonomous Avatars. Convergence, 7(2), pp.47-65. DOI: https://doi.org/10.1177/135485650100700205

30. Jennifer Radbourne, Katya Johanson and Hilary Glow. 2010. Empowering audiences to measure quality. Participations: Journal of audience \& reception studies, 7(2), pp.360-379.

31. Matthew Reason. 2010. Asking the audience: audience research and the experience of theatre. About performance (10). pp. 15-34..

32. Stuart Reeves. 2011. Designing interfaces in public settings: Understanding the role of the spectator in
Human-Computer Interaction. Springer Science \& Business Media.

33. Guy Schofield, Tom Bartindale, and Peter Wright. 2015. Bootlegger: Turning Fans into Film Crew. In Proceedings of the 33rd Annual ACM Conference on Human Factors in Computing Systems (CHI '15). ACM, New York, NY, USA, 767-776. DOI: https://doi.org/10.1145/2702123.2702229

34. Phoebe Sengers and Bill Gaver. 2006. Staying open to interpretation: engaging multiple meanings in design and evaluation. In Proceedings of the 6th conference on Designing Interactive systems (DIS '06). ACM, New York, NY, USA, 99-108.

DOI=http://dx.doi.org/10.1145/1142405.1142422

35. Jennifer G. Sheridan, Nick Bryan-Kinns, and Alice Bayliss. 2007. Encouraging witting participation and performance in digital live art. In Proceedings of the 21st British HCI Group Annual Conference on People and Computers: HCI...but not as we know it - Volume 1 (BCS-HCI '07), Vol. 1. British Computer Society, Swinton, UK, UK, 13-23.

36. John A. Sloboda, Susan A. O'Neill and Antonia Ivaldi. 2001. Functions of music in everyday life: An exploratory study using the Experience Sampling Method. Musicae scientiae, 5(1), pp.9-32. DOI: https://doi.org/10.1177/102986490100500102

37. Lucy A. Suchman. 1987. Plans and situated actions: The problem of human-machine communication. Cambridge university press.

38. Raphael Velt, Steve Benford, and Stuart Reeves. 2017. A Survey of the Trajectories Conceptual Framework: Investigating Theory Use in HCI. In Proceedings of the 2017 CHI Conference on Human Factors in Computing Systems (CHI '17). ACM, New York, NY, USA, 2091-2105. DOI: https://doi.org/10.1145/3025453.3026022

39. Bradley W. Vines, Carol L. Krumhansl, Marcelo M. Wanderley, Ioana M. Dalca, and Daniel J. Levitin. 2011. Music to my eyes: Cross-modal interactions in the perception of emotions in musical performance. Cognition, 118(2), pp.157-170. DOI: https://doi.org/10.1016/j.cognition.2010.11.010

40. David M. Weigl and Kevin R. Page. 2017. A framework for distributed semantic annotation of musical score: "Take it to the bridge!". In Proceedings of the 18th International Society for Music Information Retrieval Conference (ISMIR 2017), Suzhou, China, 221-228. 